\title{
A SEMIMARTINGALE APPROACH TO SOME PROBLEMS \\ IN RISK THEORY
}

\author{
By Michael Sørensen \\ Department of Theoretical Statistics \\ University of Aarhus \\ Ny Munkegade \\ DK-8000 Aarhus C, Denmark
}

\begin{abstract}
The purpose of this note is to draw attention to a semimartingale method which can be applied to very general types of risk models to obtain local martingales or martingales, which can then be used in the now classical way to evaluate ruin probabilities. Relations to the theory of exponential families of stochastic processes are also pointed out and utilized.
\end{abstract}

\section{INTRODUCTION}

Since Gerber (1973) introduced the use of martingale methods in risk theory, these methods have become a standard technique, see also Gerber (1979). Several papers have appeared, including Dassios and Embrechts (1989), Delbaen and Haezendonck (1989) and Schmidli (1995), where martingale methods have been used to analyse increasingly complicated risk models. A more comprehensive review of the literature can be found in Grandell (1991) and Schmidli (1994). In this note we use results from the general theory of semimartingales to derive martingales or local martingales which can be used in the now classical way to asses the probability of ruin in very general risk models.

\section{THE RUIN PROBABILITY FOR A GENERAL RISK MODEL}

In this section we will consider risk processes of the following type

$$
X_{t}=u+B_{t}+Z_{t}+S_{t}
$$

where $B_{0}=Z_{0}=S_{0}=0$ such that the constant u is the initial capital. The process $B$ represents the total premium payments between time 0 and time $t$ and the accumulation of other regular and predictable streams of income or payment. It is assumed to be a continuous process of finite variation. The process $S$ is a jump process representing the accumulated claims, while $Z$ is a random perturbation which is assumed to be a continuous local martingale. Thus $S_{t}$ is the sum of the jumps of $X$ in the time interval $[0, t]$. We assume that there exists a predictable process $S$ of finite ASTIN BULLETIN, Vol. 26, No. 1. 1996, pp. 15-23 
variation such that $S_{t}-\widetilde{S}_{t}$ is a local martingale. This is a very weak condition which usually follows from the Doob-Meyer decomposition theorem. It implies that the risk process is a semimartingale (It is, in fact, a particularly nice kind of semimartingale, which in the stochastic calculus is referred to as a special semimartingale). We will, moreover, assume that the times at which claims occur cannot be predicted. The technical way of expressing this is that we assume the process $X$ to be quasi-left-continuous. A more precise definition of this concept can, for instance, be found in Jacod and Shiryaev (1987), but for the discussion here the slightly loose definition just given is sufficient. Note that a quasi-left-continuous process is by no means required to have continuous sample paths. To state these conditions precisely, it is necessary that all processes are defined on a probability space $(\Omega, \mathcal{F}, P)$ with a right-continuous filtration $\left\{\mathcal{F}_{t}\right\}$. It is not necessary to be very precise about this here, but it may certainly be so in some applications.

The process $Z$ need not simply be some unspecific perturbation. It could, for instance, be due to the randomly varying value of a portfolio of stocks. If $A_{t}$ is the value of the portfolio at time $t$, a simple classical model for the variation of $A$ is the geometric Brownian motion $d A_{t}=\alpha A_{t} d t+\sigma A_{t} d W_{t}$, where $W$ is a Wiener process. In this case the accumulated income in $[0, t]$ from the stock portfolio $\alpha \int_{0}^{t} A_{s} d s$ is included in $B_{t}$, while $Z_{t}=\sigma \int_{0}^{t} A_{s} d W_{s}$.

We have assumed that the sum of the jumps of $X$ in $[0, t]$ is convergent. This is not the case for all semimartingales, but we make the assumption because it simplifies the exposition considerably and is satisfied for most risk models of practical interest. Note, however, that there exist results without this assumption which are similar to, but more complicated than, the following. Since $S$ represents the claims, all jumps are downwards. The assumptions imposed imply the existence of a predictable random measure $\nu(\omega ; d t, d x)$ on $(0, \infty) \times(-\infty, 0)$ satistying $\nu(\{t\} \times(-\infty, 0))=0$ almost surely for all $t>0$ and

$$
-\int_{0}^{t} \int_{-\infty}^{0} x \nu(d s, d x)<\infty
$$

almost surely for all $t>0$, such that

$$
S_{t}-\int_{0}^{t} \int_{-\infty}^{0} x \nu(d s, d x)
$$

is a local martingale, see Jacod and Shiryaev (1987, Section II.1). In the context of risk theory, $\nu$ could be called the claim intensity measure. The net-profit condition for the model (2.1) can be expressed as

$$
B_{t}>-\int_{0}^{t} \int_{-\infty}^{0} x \nu(d s, d x) \text { for all } t>0
$$


This means that the insurance company adopts the sensible policy to let the premiums follow the claim intensity. Whether this can exactly be done in practice depends on how the claim intensity varies with time.

If we make the further assumption that there exists an $r_{0}>0$ such that

$$
\int_{0}^{t} \int_{x<-1} e^{-r x} \nu(d s, d x)<\infty
$$

almost surely for all $t>0$ when $0<r \leq r_{0}$, then it follows from the general semimartingale theory that the stochastic process

$$
M_{t}(r)=\exp \left[-r\left(X_{t}-u\right)-G_{i}(r)\right]
$$

is a local martingale for every $r$ in $\left[0, r_{0}\right]$, see Liptser and Shiryaev (1989, Chapter 4). Here

$$
G_{t}(r)=-r B_{t}+\frac{1}{2} r^{2}\langle Z\rangle+\int_{0}^{t} \int_{-\infty}^{0}\left(e^{-r x}-1\right) \nu(d s, d x)
$$

and $\langle Z>$ denotes the predictable quadratic variation of $Z$.

The local martingale $M(r)$ can be used to evaluate the ruin probability in the way that is now standard in risk theory. Let

$$
\tau=\inf \left\{t>0: X_{t}<0\right\}
$$

be the time of ruin. Then because a non-negative local martingale is a supermartingale and because $M_{0}(r)=1$, it follows that

$$
1 \geq E\left(M_{\tau \wedge t}(r)\right) \geq E\left(M_{\tau}(r) \mid \tau \leq t\right) P(\tau \leq t)
$$

for every $r$ in $\left[0, r_{0}\right]$ and $t>0$. Hence

$$
P(\tau \leq t) \leq \frac{e^{-r u}}{E\left(\exp \left[-G_{\tau}(r)\right] \mid \tau \leq t\right)} \text { for all } r \in\left[0, r_{o}\right],
$$

where we have used that $X_{\tau} \leq 0$ on $\{\tau \leq t\}$; see also Gerber (1979, p. 133).

By Jensen's inequality we see that

$$
E\left(\exp \left[-G_{\tau}(r)\right] \mid \tau \leq t\right)^{-1} \leq E\left(\exp \left[G_{\tau}(r)\right] \mid \tau \leq t\right)
$$

Note that $G_{\tau}(r)$ is a strictly convex function of $r$ satisfying that $G_{\tau}(0)=0$ and

$$
-G_{\tau}^{\prime}(0)=B_{\tau}+\int_{0}^{\tau} \int_{-\infty}^{0} x \nu(d s, d x)>0
$$

by the net-profit condition (2.3). That we can differentiate the integral with respect to $\nu$ under the integral follows by a standard argument because zero is an interior point in the range of $r$-values for which the integral exists. From these observations it follows that 


$$
E\left(\exp \left[-r u+G_{\tau}(r)\right] \mid \tau \leq t\right)
$$

is a strictly convex function of $r$ which decreases from the value 1 at $r=0$. When (2.4) holds for all $r>0$, it increases to plus infinity as $r \rightarrow \infty$. Hence there exists a unique $r^{*} \in\left[0, r_{0}\right]$ for which this function attains its minimum, and by $(2.8)$

$$
P(\tau \leq t) \leq \frac{e^{-r^{*} u}}{E\left(\exp \left[-G_{\tau}\left(r^{*}\right)\right] \mid \tau \leq t\right)}
$$

is probably often close to the best evaluation of the ruin probability obtainable from (2.7).

A simpler evaluation of the ruin probability is obtained if an r-value $R_{t}>0$ exists for which the denominator of (2.7) equals one. This $r$-value need not be unique, and it typically depends on $t$. We see that

$$
P(\tau \leq t) \leq e^{-R, u} .
$$

If $R_{t}$ exists for all $t>0$ and if $R=\lim _{t \rightarrow \infty} R_{t}$ exists, then

$$
P(\tau<\infty) \leq e^{-u R}
$$

Example 2.1 Consider the classical risk model perturbed by a Wiener process $W$ :

$$
X_{t}=u+c t+\sigma W_{t}-\sum_{i=1}^{N_{t}} Y_{i}
$$

Here $c$ is the premium rate, $N$ is a Poisson process with intensity $\lambda$, and the $Y_{i}$ 's are positive independent identically distributed random variables with distribution function $F$ and mean value $\mu$. We assume that $W, N$ and $\left\{Y_{i}\right\}$ are independent. This model has been studied by Gerber (1970), Dufresne and Gerber (1991), Furrer and Schmidli (1994) and Schmidli (1995).

In this particular case, $B_{t}=c t,\langle Z\rangle_{t}=\sigma^{2} t$ and $\nu(\omega ; d t, d x)=\lambda F^{*}(d x) d t$, with $F^{*}(x)=1-F(-x)$, so

$$
G_{t}(r)=g(r) t=\left(-r c+\frac{1}{2} \sigma^{2} r^{2}+\lambda\left[\varphi_{F}(-r)-1\right]\right) t
$$

where $\varphi_{F}(s)=\int e^{-s x} d F(x)$ is the Laplace transform of $F$. Since $X$ in this case is a process with independent increments, it is well-known that $M_{t}(r)$ is a martingale for every $r$ in the domain of $\varphi_{F}$. We see that $R_{t}=R$ is the positive solution of $g(r)=0$. When $\sigma^{2}=0, R$ is the classical adjustment (or Lundberg) coefficient.

A bound on finite time ruin probabilities, which is more precise than $\exp (-R u)$, can in some cases be obtained as follows. For $r \in\left[R, r_{0}\right]$ we have that $g(r) \geq 0$, so by $(2.7)$

$$
P(\tau \leq t) \leq \exp [-r u+g(r) t] \text { for all } r \in\left[R, r_{0}\right] .
$$

The right hand side of (2.15) attains its minimum at $r^{*}$, which is given as the solution of $g^{\prime}\left(r^{*}\right)=u / t$, provided there is a solution in $\left[0, r_{0}\right]$. Otherwise the 


\subsection{Discussion}

In parallel with the previous case, this time the product term ${ }_{1} \pi_{x} a_{x}$ in the expression for $E\left(R_{x}^{\prime}\right)$ involving the unobserved number of deaths $a_{x}$ based on head counts has been replaced by $a_{x}^{\prime}$, the observed number of deaths base on policy counts. Again result (5.4b) follows trivially from result (5.4a).

A knowledge of the reciprocals of the dispersion parameters $\psi_{x}$ is required to form the weights if the distribution assumptions (5.4a or b) are to be fully implemented. In the event that the results of a study into the variance ratios for the policies in question are available, this will furnish estimates for the first two moments $\pi_{1}$ and ${ }_{2} \pi_{x}$ of the number of duplicate policies so that modelling can proceed. Alternatively if it is assumed that the square of the coefficient of variation of the number of duplicate policies held by an individual is sufficiently small so as to make the first term on the RHS of expression (5.5) for $\psi_{x}$ is negligible in comparison with the second term,

$$
\psi_{x}=\phi_{x} \frac{1}{a_{x}^{\prime}}
$$

and the situation is analogous to that discussed in Section 4.3.

\section{ILLUSTRATION}

The dual methodologies are illustrated using the Pensioners' widows 1979-1982 experience reported in Table 15.5 of Forfar et al. (1988). The data $\left(a_{x}, r_{x}\right)$, comprising the numbers of deaths $a_{x}$ and matching central exposures $r_{x}$, are reported in the age range 17 to 108 years inclusive. There are $2+5=7$ completely empty cells in the extremities of the age range and $28+12=40$ cells contain no reported deaths. The detail of the graduation contained in the above Table is based on Gompertz's formula fitted by the 'conventional' approach, in which the numbers of deaths are modelled as Poisson random variables. The data have been regraduated using both the 'conventional' approach based on assumptions (3.4a) with predictor-link formulation

$$
\log m_{x}=\log r_{x}+\log \mu_{x+1 / 2}=\log r_{x}+\beta_{0}+\beta_{1}\left(\frac{x-70}{50}\right),
$$

and the dual approach based on assumptions (3.5a) with equivalent predictor-link formulation

$$
\log m_{x}=\log a_{x}-\log \mu_{x+1 / 2}=\log a_{x}+\beta_{0}+\beta_{1}\left(\frac{x-70}{50}\right),
$$

where $m_{x}$ denotes the respective mean responses. The associated graduation formula, implied by these formulae, is taken from Forfar et al. (1988). Some details of the respective fits including the parameter estimates are recorded in Table 6.1. The corresponding parameter estimates have opposite signs as expected, but differ slightly in absolute value because the data entries involving zero deaths feature only in the 'conventional' analysis. Similarly the corresponding values of both the deviances and 
We shall now describe simple situations where the ruin probability can easily be evaluated. We suppose that for each $t>0$ there exists a distribution function $\widetilde{F}_{t}$ such that $F_{s}(x) \geq \widetilde{F}_{t}(x)$ for all $x>0$ and all $s \leq t$, i.e. the claim-size distribution at time $s$ is stochastically dominated by a single distribution $\widetilde{F}_{t}$ for all $s \leq t$. This is, for instance, the case if the claims are subject to deterministic inflation. Under the condition just imposed, $\mu_{s} \leq \tilde{\mu}_{t}$ for $s \leq t$ and

$$
\int_{0}^{s}\left[\varphi_{u}(-r)-1\right] \lambda_{u} d u \leq\left[\widetilde{\varphi}_{t}(-r)-1\right] \Lambda_{s} \text { for } s \leq t
$$
where $\widetilde{\mu}_{t}$ denotes the mean value of $\widetilde{F}_{t}, \widetilde{\varphi}_{t}(u)=\int e^{-u x} d \widetilde{F}_{t}(x)$, and $\Lambda_{s}=\int_{0}^{t} \lambda_{s} d s$ is
the integrated intensity of $N$.

Now we make the further assumption that the insurance company adopts the prudent policy that for some constant $c>1$

$$
B_{s} \geq c \tilde{\mu}_{t} \int_{0}^{s} \lambda_{u} d u
$$

for $s \leq t$. If, moreover, $\sigma_{s}^{2}$ is bounded by a constant $\zeta_{t}^{2}$ for $s \leq t,(3.4)$ and (3.5) implies that

$$
\begin{aligned}
G_{s}(r) & \leq\left[-r c \widetilde{\mu}_{t}+\widetilde{\varphi}_{t}(-r)-1\right] \Lambda_{s}+\frac{1}{2} r^{2} \zeta_{t}^{2} s \\
& =g_{t}(r) \Lambda_{s}+\frac{1}{2} r^{2} \zeta_{t}^{2} s
\end{aligned}
$$

for all $s \leq t$. The function $g_{t}(r)$ is well-known from classical risk theory. Under the conditions imposed it is convex, $g_{t}(0)=0$ and $g_{t}^{\prime}(0)<0$, so there is a range $\left[0, R_{t}\right]$ of $r$-values for which $g_{t}(r) \leq 0$. Note that $R_{t}$ is an analogue of the classical adjustment coefficient. For $r \in\left[0, r_{0}\right]$ it follows from (2.7) that

$$
P(\tau \leq t) \leq \frac{e^{-r u+\frac{1}{2} r^{2} \zeta_{t}^{2} t}}{E\left(\exp \left[-g_{t}(r) \Lambda_{\tau}\right] \mid \tau \leq t\right)} .
$$

The Laplace transform of $\Lambda_{\tau}$ is rarely known, but when the Laplace transform of $\Lambda_{t}$ is known, it is sometimes possible to proceed in a way analogous to the derivation of the upper bound (2.16). Quite generally we can use that $-r u+\frac{1}{2} r^{2} \zeta_{t}^{2} t$ has a minimum at $r=u /\left(t \zeta_{t}^{2}\right)$, which implies the inequality

$$
P(\tau \leq t) \leq e^{-\frac{1}{2} u^{2} /\left(t \zeta_{t}^{2}\right)}
$$

provided $u /\left(t \zeta_{t}^{2}\right) \leq R_{t}$. In general, we have the result

$$
P(\tau \leq t) \leq \exp \left(-R_{t} u+\frac{1}{2} R_{t}^{2} \zeta_{t}^{2} t\right)
$$

This evaluation is, of course, most precise when we can choose $R_{t}$ such that $g_{t}\left(R_{t}\right)=$ 
0 . Note that we could, in a similar way, treat the case where $\zeta_{t}^{2}$ is a random variable independent of $\Lambda_{\tau}$ provided the Laplace transform of $\zeta_{t}^{2}$ is known. Another possible and manageable assumption is that the process $\sigma^{2}$ is bounded by a constant times the intensity $\lambda$.

Finally, we make the stronger assumption that for $s \leq t$ the intensity $\lambda_{s}$ is bounded by a constant $d_{t}>0$. By (2.7)

$$
P(\tau \leq t) \leq \frac{e^{-r u}}{E\left(\exp \left[-h_{f}(r) \tau\right] \mid \tau \leq t\right)}
$$

with $h_{t}(r)=g_{t}(r) d_{t}+\frac{1}{2} r^{2} \zeta_{t}^{2}$. The right-hand side of (3.8) is of the type considered in Example 2.1, and many ideas that have been used to study that example can be applied here too. Obviously,

$$
P(\tau \leq t) \leq \exp \left(-R_{t} u\right)
$$

where $R_{t}$ is the unique strictly positive solution to $h_{t}(r)=0$. Here we use that $h_{t}(r)$ is strictly convex with $h_{t}(0)=0$ and $h_{t}^{\prime}(0)<0$ provided the strong net-profit condition (3.5) is satisfied.

\section{THE MARTINGALE CASE}

Sometimes it can be proved that $E\left(M_{t}(r)\right)=1$ for all $t \geq 0$. Then it follows that the supermartingale $M_{t}(r)$ is a martingale. We shall briefly consider this situation, where more accurate results can be obtained, see e.g. Gerber (1979) and Schmidli (1995). A nice way of seeing this, which also shows how the theory is related to the theory of exponential families of processes, is to define for each $r \in\left[0, r_{0}\right]$ a new probability measure $Q_{r}$ by

$$
Q_{r}(A)=\int_{A} M_{t}(r) d P
$$

for $A \in \mathcal{F}_{t}$ and for all $t>0$. By the fundamental identity of sequential analysis (see e.g. Küchler and Sørensen, 1994)

$$
P(\tau \in B)=E_{Q_{r}}\left(\exp \left[r X_{\tau}+G_{\tau}(r)\right] 1_{\{\tau \in B\}}\right) e^{-r u}
$$

where $B \subseteq \mathbb{R}$. The right-hand side can be evaluated as discussed earlier.

The family of probability measures $\left\{Q_{r}: 0<r \leq r_{0}\right\}$ was also studied in Sørensen (1993). Under an additional assumption on $\nu$ it is the exponential family of processes generated by the semimartingale $X$. This is, for instance, the case for the general type of models considered in Section 3 when the claim-size distribution is constant. We will now concentrate on such models. 
Under $Q_{r}$ the process $X$ is of the form

$$
X_{t}=u+\widetilde{B}_{t}+\int_{0}^{t} \sigma_{s} d \widetilde{W}_{s}-\sum_{i=1}^{N_{t}} Y_{i}
$$

where $\tilde{W}$ is a standard Wiener process, $N$ has intensity $\varphi(-r) \lambda$, the claim-size distribution is $\exp [r x-\log \varphi(-r)] d F(x)$ (i.e. it belongs to the exponential family generated by $F$ ), and

$$
\left.\widetilde{B}_{t}=B_{t}-r<Z\right\rangle_{t}
$$

All independence assumptions made under $P$ hold under $Q_{r}$ too. These results follow e.g. from Jacod and Mémin (1976), see also Küchler and Sørensen (1989).

We shall now consider the event $\{\tau<\infty\}$. The probability of this event under $Q_{r}$ is determined by the predictable drift of $X$ under $Q_{r}$, given by

$$
\begin{aligned}
\widetilde{B}_{t}-\int_{0}^{t} \int_{0}^{\infty} x \exp [r x-\log \varphi(-r)] d F(x) \varphi(-r) \lambda_{s} d s & =B_{t}-r\langle Z\rangle+\varphi^{\prime}(-r) \Lambda_{t} \\
& =-G_{t}^{\prime}(r),
\end{aligned}
$$

where we have used that, by standard exponential family theory, the mean value of the claim-size distribution under $Q_{r}$ is $-\varphi^{\prime}(-r) / \varphi(-r)$. We saw in Section 2 that under the net-profit condition $-G_{t}(r)$ is a strictly concave function of $r$ satisfying $G_{t}(0)=0$ and $-G_{t}^{\prime}(0)>0$. Now suppose the model is sufficiently ergodic that $t^{-1} G_{t}(r)$ converges almost surely to a non-random limit $g(r)$, which will then be convex. Assume further that we can find $R>0$ such that $g(R)=0$. Then $-g^{\prime}(R)<0$, so $-G_{t}^{\prime}(R)$ will tend to minus infinity as $t \rightarrow \infty$. Hence $Q_{r}(\tau<\infty)=1$ for $r \geq R$, so it follows from (4.2) that

$$
P(\tau<\infty)=E_{Q}\left(\exp \left[r X_{\tau}+G_{\tau}(r)\right]\right) e^{-r u}
$$

for $r \geq R$.

\section{REFERENCES}

Dassios, A. \& Embrechts, P. (1989). Martingales and insurance risk. Commun. Statist. Stochastic Models $5,181-217$.

Delbaen, F. \& HaEzendonck, J. (1989). A martingale approach to premium calculation principles in an arbitrage free market. Insurance Math. Econom. 8, 269-277.

Dufresne, F. \& Gerber, H.U. (1991). Risk theory for the compound Poisson process that is perturbed by diffusion. Insurance Math. Econom. 10, 51-59.

FurRer, H.J. \& SChmidLI, H. (1994). Exponential inequalities for ruin probabilities of risk processes perturbed by diffusion. Insurance Math. Econom. 15, 23-36.

GERBER, H.U. (1970). An extension of the renewal equation and its application in the collective theory of risk. Skand. Aktuar Tidskr. 53, 205-210.

GERBER, H.U. (1973). Martingales in risk theory. Schweiz. Verein. Versicherungsmath. Mitt. 73, 205-216. 
Gerber, H.U. (1979). An Introduction to Mathematical Risk Theory. Huebner Foundation Monographs, Philadelphia.

Grandell, J. (1991). Aspects of Risk Theory. Springer-Verlag, New York.

JACOD, J. \& MÉmIN, J. (1976). Caractéristiques locales et conditions de continuité absolue pour les semimartingales. Z. Wahr. verv. Geb. 35, 1-37.

JaCod, J. \& Shiryaev, A.N. (1987). Limit Theorems for Stochastic Processes. Springer-Verlag, Heidelberg.

Küchler, U. \& Sørensen, M. (1989). Exponential families of stochastic processes: A unifying semimartingale approach. Internat. Statist. Rev. 57, 123-144.

KüCHLER, U. \& SøRENSEN, M. (1994): Exponential families of stochastic processes and Lévy processes. J. Statist. Plann. Inference 39, 211-237.

Liptser, R.Sh. \& ShiryaeV, A.N. (1989). Theory of Martingales. Kluwer Academic Publishers, Dordrecht.

SCHMIDLI, H. (1994). Martingales and insurance risk. Research Report 289, Dept. Theor. Statist., Univ. of Aarbus.

SChmidli, H. (1995). Cramér-Lundberg approximations for ruin probabilities of risk processes perturbed by diffusion. Insurance Math. Econom. 16, 135-149.

SøRENSEN, M. (1993). The exponential family generated by a semimartingale. Research Report 269 , Dept. Theor. Statist., Univ. of Aarhus. To appear in Proceedings of the Fourth Finnish-Russian Symposium on Probability Theory and Mathematical Statistics. 